\title{
Lysosome Sensing Is a Key Mechanism in Leishmania Intracellular Development
}

\author{
Dan Zilberstein* \\ Faculty of Biology, Technion - Israel Institute of Technology, Haifa, Israel
}

Phagolysosomes of macrophages are the niche where the parasitic protozoan Leishmania resides and causes human leishmaniasis. During infection, this organism encounters dramatic environmental changes. These include heat shock (from $26^{\circ} \mathrm{C}$ in the vector to $33^{\circ} \mathrm{C}$ or $37^{\circ} \mathrm{C}$ in the host, for cutaneous and visceral species, respectively) and acidic $\mathrm{pH}$ typical to the lysosome and nutrient availability. Leishmania cells developed ways to sense the lysosome-specific environment (acidic $\mathrm{pH}$ and body temperature) as means of recognition and, subsequently, initiation of differentiation into the intracellular form. Recent studies have indicated that protein kinase A plays a role as

OPEN ACCESS

Edited by: Annette Elizabeth Kaiser, University of Duisburg-Essen, Germany

Reviewed by: Maria Fernanda Laranjeira-Silva, University of São Paulo, Brazil Angamuthu Selvapandiyan, Jamia Hamdard University, India

*Correspondence:

Dan Zilberstein danz@technion.ac.il

Specialty section:

This article was submitted to Antimicrobials, Resistance and Chemotherapy,

a section of the journal

Frontiers in Microbiology

Received: 14 February 2021

Accepted: 25 March 2021

Published: 07 May 2021

Citation:

Zilberstein D (2021) Lysosome Sensing Is a Key Mechanism in Leishmania Intracellular

Development.

Front. Microbiol. 12:667807. doi: 10.3389/fmicb.2021.667807 the gatekeeper that enables differentiation initiation. This review provides an update on the lysosome signaling pathway-mediated Leishmania intracellular development.

Keywords: Leishmania, development, sensing, differentiation, macrophages

\section{INTRODUCTION}

Pathogenic microorganisms invade and colonize our body because it contains nutrients that pathogens need to complete their developmental cycle. Following infection, the microorganisms must quickly find their destinations, which is where the specific nutrients can be reached. Over many years of "learning", these pathogens have selected host elements that are unique and thereby tag these locations. Parasites developed sensors that utilize these cues as ligands that indicate their arrival at their destination. Once they colonize, a second line of sensors monitor metabolic availability inside that compartment, enabling parasite to manipulate and survive host harsh conditions (Mancio-Silva et al., 2017; Zilberstein, 2018; Landfear and Zilberstein, 2019). My research group has investigated these processes using Leishmania as a model organism.

Leishmania is an intracellular parasite that cycles between the midgut of female sand flies and phagolysosomes of mammalian macrophages. Interestingly, Leishmania turned the extremely harsh environment inside the host macrophage into a shelter to most likely hide from the host immune system (Chang and Dwyer, 1976; Moradin and Descoteaux, 2012). To reach and identify the phagolysosomes, Leishmania developed means to sense lysosome-specific cues. Once it identifies its location inside phagolysosome, Leishmania starts to transform to the intracellular form, the amastigote. To block parasite invasion, macrophages activate means to kill them, including release of reactive oxygen species (ROS) and synthesis of cytotoxic nitric oxide (NO) by NO synthase (iNOS). The latter requires a massive conversion of arginine to NO, which exhausts intracellular arginine. To protect themselves, parasites developed sensing mechanism that monitor arginine in the phagolysosome, a mechanism that is essential for their survival and ability to develop into amastigotes (Goldman-Pinkovich et al., 2020). 
Over almost three decades, my laboratory has investigated the signaling pathways that initiate Leishmania intracellular development. We developed a host-free experimental system that enabled monitoring of promastigote-to-amastigote differentiation in axenic conditions (Barak et al., 2005; Zilberstein, 2020). Using this system, we have identified parasite molecules that helped complete the puzzle of Leishmania development inside its host macrophage. This review is an update on this puzzle, focusing on acidic $\mathrm{pH} /$ high temperature-mediated differentiation. This time, I decided to tell the story with a flavor of historical perspective.

\section{Intracellular Leishmania Develops Inside Mammalian Phagolysosomes: What Does It Physiologically Mean?}

It became apparent from studies in the 1970s that Leishmania differentiation from extracellular promastigote to intracellular amastigotes occurs within the phagolysosome of macrophages (Alexander and Vickerman, 1975; Chang and Dwyer, 1976; Berman et al., 1979). The in vitro studies were corroborated by the in vivo demonstration that when BALB/C mice footpads, infected by Leishmania, are suspended in a solution containing colloidal gold, the gold particles colocalized with amastigotes in the phagolysosomes of foot pad macrophages (Berman et al., 1981). These studies determined that phagocytosis is the basic mechanism underlying Leishmania entry into the host, but concomitant with some active input by parasites (Zilberstein and Shapira, 1994; Antoine et al., 1998; Lodge and Descoteaux, 2008).

Exactly how parasites attach to macrophages and what are the molecules that form the invasion pathway are still not fully known, even though progress has been made (Rosazza et al., 2020; Smirlis et al., 2020). A recent review has summarized genetic and biochemical details on the molecular mechanism of macrophage invasion (Horta et al., 2020). Our focus is on Leishmania-regulated formation of the acidic phagolysosomes and their role in the initiation of promastigote differentiation into amastigotes. During phagocytosis of uninfected macrophages, it takes about 30 min for lysosome markers to appear in a phagosome, whereas it takes more than $2 \mathrm{~h}$ for lysosome markers to appear in promastigote-infected phagosomes (Antoine et al., 1998; Scianimanico et al., 1999; Vinet et al., 2009; Da Silva Vieira et al., 2019). Lipophosphoglycan (LPG), a major component of the promastigote surface, plays a key role in the infective pathway as it restricts transiently the fusion of nascent promastigotecontaining phagosomes with late endocytic compartments (Desjardins and Descoteaux, 1997; Moradin and Descoteaux, 2012). Interestingly, LPG delays the appearance of vacuolar ATPases in phagosome membranes until they fuse with primary lysosomes. Hence, LPG delays phagosome acidification and maturation (Vinet et al., 2009). This is critical because, as explained later, sensing acidic $\mathrm{pH}$ is one of the two cues that signal parasite arrival to the phagolysosome and subsequently initiate promastigote differentiation into amastigotes (Zilberstein and Shapira, 1994; Saar et al., 1998; Zilberstein, 2008).
Mutant promastigotes that lack LPG are delivered quicker to lysosomes and, consequently, are more susceptible to macrophage killing than wild-type promastigotes (Scianimanico et al., 1999; Spath et al., 2000; Turco et al., 2001; Moradin and Descoteaux, 2012). However, it is agreed by most experts in the field that LPG is not the only factor that regulates Leishmania entry into the host (Horta et al., 2020). For example, LPG null mutants of Leishmania mexicana are as effective as wild type in macrophage invasion (Ilg, 2000). The demonstration that virulent Leishmania chagasi is localized in caveolae during phagocytosis by host macrophages has prompted speculation that these specialized membrane domains play a role in intracellular parasite survival by targeting parasites to a phagocytosis pathway where lysosome fusion is delayed (Rodriguez et al., 2006). These analyses further support the notion that metacyclic-specific LPG delays the process of phagosome maturation until parasite complete shedding it. Hence, everything that happens after the pause is independent of LPG. Based on this model for the invasion pathway, I proposed that parasites that reach the acidic phagolysosomes and start to differentiate to amastigotes are not metacyclic anymore (Zilberstein, 2008).

To summarize the preceding section, phagolysosome biogenesis in Leishmania-infected macrophages is actively delayed by the invading parasite to assure that they do not initiate transformation into amastigotes before LPG is released and phagolysosome completed maturation.

\section{Sensing the Lysosome-Specific Environment Is How Leishmania Identifies Its Intracellular Destination}

Once engulfed into the macrophage phagosome, except for shedding LPG, promastigotes halt development until phagosomes fuse with primary lysosomes to form the acidic phagolysosomes. Parasites that not long ago underwent heat shock $\left(33^{\circ} \mathrm{C}\right.$ and $37^{\circ} \mathrm{C}$ for cutaneous and visceral species, respectively) are now, in addition, exposed to acidic $\mathrm{pH}$ (around 5.5; Courret et al., 2001; Séguin and Descoteaux, 2016). Parasite cells combine these two cues into a single signaling pathway that indicates arrival at their destination and thereby initiates promastigote differentiation into amastigotes. However, this is not a straightforward phenomenon. It was important to show that exposing parasites concomitantly to these two stress conditions indeed activates a true signal transduction pathway, not an additional stress response.

Experiments have been carried out to prove that concomitant exposure to acidic $\mathrm{pH}$ and high temperature signal promastigotes to start differentiating into amastigotes. Exposing axenic promastigotes to either acidic $\mathrm{pH}$ without changing the temperature (Zilberstein et al., 1991) or to $37^{\circ} \mathrm{C}$ without changing the medium $\mathrm{pH}(\mathrm{Pan}, 1984)$ induced expression of a few amastigote-specific genes, but parasites did not differentiate. Only combining both acidic $\mathrm{pH}$ and high temperature induced promastigote differentiation into amastigotes (Bates, 1994; Saar et al., 1998; Zilberstein, 2020). Barak et al. (2005) exposed promastigotes to $\mathrm{pH} 5.5$ but replaced the high temperature with $5 \%$ methanol or $200 \mathrm{mM}$ of azetidine-2-carboxylic acid, 
a synthetic proline analog. These compounds induce protein misfolding, typical to heat shock response. Leishmania donovani promastigotes grown in these conditions started to differentiate as did parasites that were exposed to the complete differentiation signal. The stronger evidence for the nature of the differentiation signal came from a phosphoproteomic experiment we have carried out in L. donovani promastigotes that were exposed for $2.5 \mathrm{~h}$ to $\mathrm{pH} 5.5$ at $26^{\circ} \mathrm{C}, \mathrm{pH} 7$ at $37^{\circ} \mathrm{C}$, or both, i.e., $\mathrm{pH} 5.5$ at $37^{\circ} \mathrm{C}$. The analysis revealed protein phosphorylation induced by either acidic $\mathrm{pH}$ or temperature. But most interestingly, these analyses discovered phosphorylation that was induced only by the complete signal (see figure 6 in Tsigankov et al., 2014). Eight such proteins were identified: two proteins of the translation machinery, two unknown protein kinases, and four hypothetical proteins. Altogether, these studies indicated that the combined $\mathrm{pH} 5.5$ and $37^{\circ} \mathrm{C}$ is a true signal that activates a signal transduction pathway in promastigotes that initiate differentiation into amastigotes.

To further demonstrate that the differentiation signal induces a true signaling pathway, Rosenzweig et al. (2008) performed a proteomic time course analysis along L. donovani differentiation. In those days, the time affinity tagging of peptide started to merge for quantitative proteomics. My laboratory was one of the first to apply isobaric tags for relative and absolute quantitation (iTRAQ) to determine protein dynamics in differentiation. Promastigotes were exposed to the differentiation signal, and samples were collected at various time points along the $120 \mathrm{~h}$ of differentiation. These studies indicated that exposing promastigotes to the differentiation signal induced coordinated changes in protein abundance, including enzymes of metabolic pathways and proteins of the translation machinery (see figure 5 in Rosenzweig et al., 2008). For example, glycolytic enzyme of the cytosol gradually decreased with time as did proteins of translation pathways. In contrast, enzymes of the $\beta$ oxidation pathway and amino acid catabolism gradually increased in abundance. For the first time, these iTRAQ analyses enabled to quantitate abundance changes of more $>1,700$ proteins at the same time point. The results of these experiments indicated that the axenic differentiation represents a highly coordinated and regulated process, a phenomenon typical to a true pathway. Further transcriptomic analyses indicated that differentiation activated dynamic changes in mRNA abundance, which suggested that at the beginning of differentiation, there were more proteins whose expression is regulated by mRNA, but later, most changes were post translational in nature (Lahav et al., 2011). In my opinion, this set of experiments clearly indicates that the differentiation signal induced a true signaling pathway that initiates promastigote differentiation into amastigotes.

Development of host-free systems using axenic parasites has enabled a better understanding of the molecular mechanism of Leishmania intracellular development. L. donovani differentiation can be induced by exposing promastigotes to high temperature and acidity $\left(37^{\circ} \mathrm{C}, \mathrm{pH} 5.5,5 \% \mathrm{CO}_{2}\right)$ typically found in the phagolysosome (Zilberstein, 2020). Differentiation to mature amastigotes takes 5 days, resembling the time it takes in vivo (Courret et al., 2001). When mature axenic amastigotes are transferred into promastigote medium and incubate at $26^{\circ} \mathrm{C}$, they differentiate back to promastigotes. It takes $48 \mathrm{~h}$ for wild-type $L$. donovani to differentiate into mature promastigotes (Bachmaier et al., 2016). This flexibility of developing back and forth enables complete control of in vitro analysis of the complete developmental cycle of $L$. donovani.

Barak et al. (2005) described differentiation time course and showed that following exposure to the signal, parasite cells undergo cell cycle arrest at G1. Subsequently, differentiation continues synchronously. Morphogenesis from elongated to round cells is initiated at early hours. At $12 \mathrm{~h}$ after exposure to the differentiation, signal parasites complete rounding; and at $24 \mathrm{~h}$, they lose their flagella completely.

\section{Protein Kinase A Is the Differentiation Gatekeeper}

Among the earliest events during promastigote-to-amastigote differentiation in Leishmania donovani are changes in the phosphorylation of a yet unexplored regulatory subunit of protein kinase A (LdPKAR3, LinJ.34.2680; Tsigankov et al., 2014). LdPKAR3 exists only in the genomes of intracellular trypanosomatids that include amastigotes in their life cycle, i.e., Leishmania and American trypanosomiasis (Trypanosoma cruzi). Functionally, promastigotes contain several phosphorylated proteins with PKA-specific motifs, most of them promptly dephosphorylate after initiating promastigote-to-amastigote differentiation (Bachmaier et al., 2016). These data pointed to the involvement of a PKA pathway in Leishmania development. My laboratory hypothesized that a check point keeps promastigotes from spontaneously transforming into amastigotes. Prompt activation of PKA dissociation opens this checkpoint, thereby initiating differentiation.

Protein kinase A is ubiquitous in eukaryotic cells, where it has been implicated in regulation of growth, development, and metabolism. The catalytic subunits of PKA assemble with the regulatory subunits into a holoenzyme complex that is inactive in the absence of cyclic AMP (cAMP). Two cyclic nucleotidebinding domains (cNBDs) in each PKAR bind cAMP and thereby cause a conformational change that leads to the dissociation of the PKAC-R complex. This unbound PKAC becomes active (Taylor et al., 2012).

Trypanosomatid PKAs differ from that of higher eukaryotes (Bachmaier and Boshart, 2013; Bachmaier et al., 2019); their genomes encode a regulatory subunit, PKAR1 (LinJ.13.0160 in Leishmania), which lacks the conserved dimerization/docking domain at the $\mathrm{N}$-terminus but keeps the (pseudo) substrate inhibitor motif in the hinge and two cyclic nucleotide-binding domains at the $\mathrm{C}$ terminus. This ancient PKAR1 cannot dimerize, and therefore, the holoenzyme is a heterodimer, not heterotetrameric, as that of the higher eukaryotes. However, more interestingly, trypanosomatid PKA is a cAMP-independent protein kinase. Two essential arginines in their cNBD-binding pockets contain other amino acids (Bubis et al., 2018; Bachmaier et al., 2019). Evolutionarily, this indicates that PKAR underwent significant changes in higher single-cell Eukaryotes (i.e., plasmodium and yeast) until it became a cAMP-dependent protein kinase. This hypothesis is supported by the fact that 
genomes of the trypanosomatid family lack the G-trimeric proteins (Ivens et al., 2005; Landfear and Zilberstein, 2019). These findings indicate that the older versions of PKAR in which trypanosomatids regulate PKAC activities are different from the canonical PKA.

In addition to R1, Leishmania genome encodes LdPKAR3 (LinJ.34.2680) that similar to R1 form heterodimers with LdPKAC subunits and is cAMP-independent. In LdPKAR3, the C-terminus half is conserved with higher Eukaryotes while the N-terminus half is divergent and most likely unstructured (Fischer-Weinberger et al., 2021).

A salient feature of the R3 subunit is a set of 12 phosphorylation sites that dynamically change during differentiation (Tsigankov et al., 2013, 2014). Of these, there was a four-fold increase in the phosphorylation of serine 262 (S262) within minutes of exposure to the promastigote-to-amastigote differentiation signal. Phosphorylation of this site is also achieved by exposure to acidic $\mathrm{pH}$ only, supporting an idea that this phosphorylation is induced by a $\mathrm{pH}$ sensor that activates a downstream kinase that subsequently phosphorylates S262. This site localizes within a region of LdPKAR3 that is likely to interact with a catalytic subunit (LdPKAC).

Leishmania donovani (as do all Leishmania species) has three distinct catalytic subunits (LdPKAC1, LdPKAC2, and LdPKAC3, encoded by LinJ.35.4060, LinJ.35.4010, and LinJ.18.1090, respectively). Extensive phylogenetic analyses indicated that LdPKAC3 is conserved across all taxa, while C1 and C2 are restricted to the Kinetoplastidae. Recent experiments indicate that LdPKAR3 is covalently bound to the subpellicular microtubules at the cell cortex. R3 associates with C3, and this association is important for the elongated shape of promastigotes (Fischer-Weinberger et al., 2021).

Cumulatively, Leishmania promastigotes have the means to sense the lysosome environment, and together with heat shock response, they turn these cues to a signal that initiates differentiation. Moreover, to date, data support the idea that Leishmania PKA plays a role in transducing promastigote-to-amastigote differentiation signal, thereby initiating differentiation.

\section{Iron Metabolism Activates an Alternative Leishmania Differentiation Pathway}

Even though this review focuses on acidic $\mathrm{pH} /$ high temperatureinduced differentiation, it is not the only pathway described to date. It is established that stress, mostly deprivation of essential metabolites, triggers parasites to escape to a life form that is less susceptible to these stresses. Amastigotes are "stress-relaxed" organisms. For example, heat (Pan, 1984), overflow of serum (Doyle et al., 1991), and osmotic shock (Blum and Balber, 1996) impose shape change in promastigotes.

\section{REFERENCES}

Alexander, J., and Vickerman, K. (1975). Fusion of host cell secondary lysosomes with the parasitophorous vacuoles of Leishmania mexicana-infected macrophages. J. Protozool. 22, 502-508. doi: 10.1111/j.1550-7408.1975. tb05219.x
None of these stresses induced complete differentiation of promastigotes into amastigotes, except for the stress induced by iron. The laboratory of Norma Andrews has investigated iron transport and metabolism in Leishmania and its role in virulence and development. They found a novel role for iron uptake in orchestrating the differentiation of amastigotes, through a mechanism that involves production of ROS (Mittra and Andrews, 2013) and is independent from $\mathrm{pH}$ and temperature changes. ROS are generally thought to be deleterious for pathogens, but it is becoming increasingly apparent that they can also function as signaling molecules regulating Leishmania differentiation, in a process that is tightly controlled by iron availability. These studies indicated that the ability to import iron is critical for both promastigotes and amastigotes viability. Interestingly, Leishmania ferric iron reductase 1 (LFR1) overexpression induced differentiation into amastigotelike parasites (Rocco-Machado et al., 2019). These cells resembled intracellular amastigotes as they are round, lost most of their flagella, and are virulent. How this iron-dependent pathway functions in disease development is still to be investigated.

\section{CONCLUDING REMARKS}

In 2001, Burchmore and Barrett published an article titled "Life in vacuoles - nutrient acquisition by Leishmania amastigotes" where they described what was known almost 20 years ago on how Leishmania parasite metabolically co-op with the extreme environment inside the phagolysosome (Burchmore and Barrett, 2001). The focus was on how nutrient transport and metabolism are influenced by the acidic $\mathrm{pH}$ environment encountered by amastigotes. A few years earlier, Shapira and Zilberstein speculated that acidic $\mathrm{pH}$ and high temperature are key elements in signaling promastigotes' arrival at their destination and to employ means of adaptation to the phagolysosome milieu. Our laboratory is getting close to describe the first amino acid-sensing signal transduction pathway. This pathway will open new avenues to understand intracellular parasitism.

\section{AUTHOR CONTRIBUTIONS}

The author confirms being the sole contributor of this work and has approved it for publication.

\section{FUNDING}

Funding was provided by a grant from the German-Israel Foundation number I-112-416.10-2017. 
Doerig, C. S. Spaeth, M. Wiese, and P. M. Selzer (Hoboken, NJ: WileyBlackwell).

Bachmaier, S., Volpato Santos, Y., Kramer, S., Githure, G. B., Klöckner, T., Pepperl, J., et al. (2019). Nucleoside analogue activators of cyclic AMP-independent protein kinase A of Trypanosoma. Nat. Commun. 10:1421. doi: 10.1038/s41467019-09338-z

Bachmaier, S., Witztum, R., Tsigankov, P., Koren, R., Boshart, M., and Zilberstein, D. (2016). Protein kinase A signaling during bidirectional axenic differentiation in Leishmania. Int. J. Parasitol. 46, 75-82. doi: 10.1016/j.ijpara.2015.09.003

Barak, E., Amin-Spector, S., Gerliak, E., Goyard, S., Holland, N., and Zilberstein, D. (2005). Differentiation of Leishmania donovani in host-free system: analysis of signal perception and response. Mol. Biochem. Parasitol. 141, 99-108. doi: 10.1016/j.molbiopara.2005.02.004

Bates, P. A. (1994). Complete developmental cycle of Leishmania mexicana in axenic culture. Parasitology 108(Pt .1), 1-9. doi: 10.1017/ s0031182000078458

Berman, J. D., Fioretti, T. B., and Dwyer, D. M. (1981). In vivo and in vitro localization of Leishmania within macrophage phagolysosomes: use of colloidal gold as a lysosomal label. J. Protozool. 28, 239-242. doi: 10.1111/j.1550-7408. 1981.tb02839.x

Berman, J. D., Wyler, D. J., and Dwyer, D. M. (1979). Multiplication of Leishmania in human macrophages in vitro. Infect.Immun. 26, 375-379. doi: 10.1128/iai. 26.1.375-379.1979

Blum, J. J., and Balber, A. E. (1996). Osmotic and metabolic-induced changes in light scattering of Leishmania donovani as measured by flow cytometry. J. Eukaryot. Microbiol. 43, 213-217. doi: 10.1111/j.1550-7408. 1996.tb01393.x

Bubis, J., Martinez, J. C., Calabokis, M., Ferreira, J., Sanz-Rodriguez, C. E., Navas, V., et al. (2018). The gene product of a Trypanosoma equiperdum ortholog of the cAMP-dependent protein kinase regulatory subunit is a monomeric protein that is not capable of binding cyclic nucleotides. Biochimie 146, 166-180. doi: 10.1016/j.biochi.2017.12.010

Burchmore, R. J., and Barrett, M. P. (2001). Life in vacuoles-nutrient acquisition by Leishmania amastigotes. Int. J. Parasitol. 31, 1311-1320. doi: 10.1016/s00207519(01)00259-4

Chang, K. P., and Dwyer, D. M. (1976). Multiplication of a human parasite (Leishmania donovani) in phagolysosomes of hamster macrophages in vitro. Science 193, 678-680. doi: 10.1126/science.948742

Courret, N., Frehel, C., Prina, E., Lang, T., and Antoine, J. C. (2001). Kinetics of the intracellular differentiation of Leishmania amazonensis and internalization of host MHC molecules by the intermediate parasite stages. Parasitology 122, 263-279. doi: 10.1017/s0031182001007387

Da Silva Vieira, T., Arango Duque, G., Ory, K., Gontijo, C. M., Soares, R. P., and Descoteaux, A. (2019). Leishmania braziliensis: strain-specific modulation of phagosome maturation. Front. Cell. Infect. Microbiol. 9:319. doi: 10.3389/fcimb. 2019.00319

Desjardins, M., and Descoteaux, A. (1997). Inhibition of phagolysosomal biogenesis by the Leishmania lipophosphoglycan. J. Exp. Med. 185, 2061-2068. doi: 10.1084/jem.185.12.2061

Doyle, P. S., Engel, J. C., Pimenta, P. F., Da Silva, P. P., and Dwyer, D. M. (1991). Leishmania donovani: long-term culture of axenic amastigotes at 37 degrees $\mathrm{C}$. Exp. Parasitol. 73, 326-334. doi: 10.1016/0014-4894(91)90104-5

Fischer-Weinberger, R., Bachmaier, S., Dandugudumula, R., Phan, I. A., Almoznino, M., Githur, G. B., et al. (2021). A divergent protein kinase A in the human pathogen Leishmania is associated with developmental morphogenesis. bioRxiv. doi: 10.1101/2021.04.24.440790

Goldman-Pinkovich, A., Kannan, S., Nitzan-Koren, R., Puri, M., Pawar, H., BarAvraham, Y., et al. (2020). Sensing host arginine is essential for Leishmania parasites' intracellular development. MBio 11:e02023-20.

Horta, M. F., Andrade, L. O., Martins-Duarte, ÉS., and Castro-Gomes, T. (2020). Cell invasion by intracellular parasites - the many roads to infection. J. Cell Sci. 133:jcs232488. doi: $10.1242 /$ jcs. 232488

Ilg, T. (2000). Lipophosphoglycan is not required for infection of macrophages or mice by Leishmania mexicana. EMBO J. 19, 1953-1962. doi: 10.1093/emboj/19. 9.1953

Ivens, A. C., Peacock, C. S., Worthey, E. A., Murphy, L., Aggarwal, G., Berriman, M., et al. (2005). The genome of the kinetoplastid parasite, Leishmania major. Science 309, 436-442. doi: 10.1126/science.1112680
Lahav, T., Sivam, D., Volpin, H., Ronen, M., Tsigankov, P., Green, A., et al. (2011). Multiple levels of gene regulation mediate differentiation of the intracellular pathogen Leishmania. FASEB J. 25, 515-525. doi: 10.1096/fj.10-157529

Landfear, S. M., and Zilberstein, D. (2019). Sensing what's out there - kinetoplastid parasites. Trends Parasitol. 35, 274-277. doi: 10.1016/j.pt.2018.12.004

Lodge, R., and Descoteaux, A. (2008). Leishmania invasion and phagosome biogenesis. Subcell. Biochem. 47, 174-181. doi: 10.1007/978-0-387-78267-6_14

Mancio-Silva, L., Slavic, K., Grilo Ruivo, M. T., Grosso, A. R., Modrzynska, K. K., Vera, I. M., et al. (2017). Nutrient sensing modulates malaria parasite virulence. Nature 547, 213-216. doi: 10.1038/nature23009

Mittra, B., and Andrews, N. W. (2013). IRONy OF FATE: role of iron-mediated ROS in Leishmania differentiation. Trends Parasitol. 29, 489-496. doi: 10.1016/ j.pt.2013.07.007

Moradin, N., and Descoteaux, A. (2012). Leishmania promastigotes: building a safe niche within macrophages. Front. Cell Infect. Microbiol. 2:121. doi: 10.3389/ fcimb.2012.00121

Pan, A. A. (1984). Leishmania mexicana?: serial cultivation of intracellular stages in a cell-free medium. Exp. Parasitol. 58, 72-80. doi: 10.1016/0014-4894(84) 90022-5

Rocco-Machado, N., Cosentino-Gomes, D., Nascimento, M. T., Paes-Vieira, L., Khan, Y. A., Mittra, B., et al. (2019). Leishmania amazonensis ferric iron reductase (LFR1) is a bifunctional enzyme: unveiling a NADPH oxidase activity. Free Radic. Biol. Med. 143, 341-353. doi: 10.1016/j.freeradbiomed.2019.08.026

Rodriguez, N. E., Gaur, U., and Wilson, M. E. (2006). Role of caveolae in Leishmania chagasi phagocytosis and intracellular survival in macrophages. Cell Microbiol. 8, 1106-1120. doi: 10.1111/j.1462-5822.2006.00695.x

Rosazza, T., Lecoeur, H., Blisnick, T., Moya-Nilges, M., Pescher, P., Bastin, P., et al. (2020). Dynamic imaging reveals surface exposure of virulent Leishmania amastigotes during pyroptosis of infected macrophages. J. Cell Sci. 134, jcs 242776 . doi: $10.1242 /$ jcs. 242776

Rosenzweig, D., Smith, D., Opperdoes, F., Stern, S., Olafson, R. W., and Zilberstein, D. (2008). Retooling Leishmania metabolism: from sand fly gut to human macrophage. FASEB J. 22, 590-602. doi: 10.1096/fj.07-9254com

Saar, Y., Ransford, A., Waldman, E., Mazareb, S., Amin-Spector, S., Plumblee, J., et al. (1998). Characterization of developmentally-regulated activities in axenic amastigotes of Leishmania donovani. Mol. Biochem. Parasitol. 95, 9-20. doi: 10.1016/s0166-6851(98)00062-0

Scianimanico, S., Desrosiers, M., Dermine, J. F., Meresse, S., Descoteaux, A., and Desjardins, M. (1999). Impaired recruitment of the small GTPase rab7 correlates with the inhibition of phagosome maturation by Leishmania donovani promastigotes. Cell Microbiol. 1, 19-32. doi: 10.1046/j.1462-5822. 1999.00002.x

Séguin, O., and Descoteaux, A. (2016). Leishmania, the phagosome, and host responses: the journey of a parasite. Cell. Immunol. 309, 1-6. doi: 10.1016/j. cellimm.2016.08.004

Smirlis, D., Dingli, F., Pescher, P., Prina, E., Loew, D., Rachidi, N., et al. (2020). SILAC-based quantitative proteomics reveals pleiotropic, phenotypic modulation in primary murine macrophages infected with the protozoan pathogen Leishmania donovani. J. Proteom. 213:103617. doi: 10.1016/j.jprot. 2019.103617

Spath, G. F., Epstein, L., Leader, B., Singer, S. M., Avila, H. A., Turco, S. J., et al. (2000). Lipophosphoglycan is a virulence factor distinct from related glycoconjugates in the protozoan parasite Leishmania major. Proc. Natl. Acad. Sci. U.S.A. 97, 9258-9263. doi: 10.1073/pnas.160257897

Taylor, S. S., Ilouz, R., Zhang, P., and Kornev, A. P. (2012). Assembly of allosteric macromolecular switches: lessons from PKA. Nat. Rev. Mol. Cell Biol. 13, 646-658. doi: 10.1038/nrm3432

Tsigankov, P., Gherardini, P. F., Helmer-Citterich, M., Spaeth, G. F., Myler, P. J., and Zilberstein, D. (2014). Regulation dynamics of Leishmania differentiation: deconvoluting signals and identifying phosphorylation trends. Mol. Cell Proteom. 13, 1769-1786. doi: 10.1074/mcp.M114.037705

Tsigankov, P., Gherardini, P. F., Helmer-Citterich, M., Spath, G. F., and Zilberstein, D. (2013). Phosphoproteomic analysis of differentiating Leishmania parasites reveals a unique stage-specific phosphorylation motif. J. Proteome Res. 12, 3405-3412. doi: 10.1021/pr4002492

Turco, S. J., Spath, G. F., and Beverley, S. M. (2001). Is lipophosphoglycan a virulence factor? A surprising diversity between Leishmania species. Trends Parasitol. 17, 223-226. doi: 10.1016/s1471-4922(01)01895-5 
Vinet, A. F., Fukuda, M., Turco, S. J., and Descoteaux, A. (2009). The Leishmania donovani lipophosphoglycan excludes the vesicular proton-ATPase from phagosomes by impairing the recruitment of synaptotagmin V. PLoS Pathog. 5:e1000628. doi: 10.1371/journal.ppat.1000628

Zilberstein, D. (2008). "Physiological and biochemical aspects of leishmania develpment," in Leishmania After The genome: Biology and Control, eds P. J. Myler and N. Fasel (New York, NY: Horizon Scientific Press), 107-122.

Zilberstein, D. (2018). "Nutrient transport and sensing as pharmacological targets for leishmaniasis," in Drug Discovery for Leishmaniasis, eds L. Rivas and G. Carmen (London: The Royal Society of Chemistry), 282-296. doi: 10.1039/ 9781788010177

Zilberstein, D. (2020). In vitro culture for differentiation simulation of Leishmania spp. Methods Mol. Biol. 2116, 39-47. doi: 10.1007/978-1-0716-0294-2_3

Zilberstein, D., and Shapira, M. (1994). The role of $\mathrm{pH}$ and temperature in the development of Leishmania parasites. Annu. Rev. Microbiol. 48, 449-470. doi: 10.1146/annurev.mi.48.100194.002313
Zilberstein, D., Blumenfeld, N., Liveanu, V., Gepstein, A., and Jaffe, C. L. (1991). Growth at acidic $\mathrm{pH}$ induces an amastigote stage-specific protein in Leishmania promastigotes. Mol. Biochem. Parasitol. 45, 175-178. doi: 10.1016/ 0166-6851(91)90040-d

Conflict of Interest: The author declares that the research was conducted in the absence of any commercial or financial relationships that could be construed as a potential conflict of interest.

Copyright (c) 2021 Zilberstein. This is an open-access article distributed under the terms of the Creative Commons Attribution License (CC BY). The use, distribution or reproduction in other forums is permitted, provided the original author(s) and the copyright owner(s) are credited and that the original publication in this journal is cited, in accordance with accepted academic practice. No use, distribution or reproduction is permitted which does not comply with these terms. 